\title{
3D PCB Designing of Protecting Circuit Using Fusion 360
}

\author{
R.Sreedhar \\ Vel Tech Rangarajan \\ Dr. Sagunthala R\&D Institute of Science and \\ Technology \\ Avadi Chennai 600 062. India. \\ rsreedhareeeped@gmail.com \\ Pawan Kumar Jaiswal \\ Vel Tech Rangarajan \\ Dr. Sagunthala R\&D Institute of Science and \\ Technology \\ Avadi Chennai 600 062. India.
}

\author{
Rishab Mahato \\ Vel Tech Rangarajan \\ Dr. Sagunthala $R \& D$ Institute of Science and \\ Technology \\ Avadi Chennai 600 062. India.
}

\author{
Adarsh Chaturvedi \\ Vel Tech Rangarajan \\ Dr. Sagunthala R\&D Institute of Science and \\ Technology \\ Avadi Chennai 600 062. India.
}

\begin{abstract}
Every electronics equipment is manufactured with a single or multiple number of Printed Circuit Boards (PCB). The PCBs hold the ICs and other components and implement the interconnections between them. Compact devices can be manufactured by using 3D PCB design. Even though lot of software's available for 3D PCB design, Fusion 360 has unique merits like ease of design procedure, compact size, applicable to all size PCBs etc., In this paper step by step procedure to design a 3D model PCB layout for a protecting circuit using Fusion 360 software is discussed. This model can be used to design the complete product in a Computer Aided Design (CAD) software.
\end{abstract}

Keywords-Computer Aided Design (CAD), Fusion 360, Printed Circuit Board (PCB), PCB Layout, PCB Routing, 3D Modelling.

\section{INTRODUCTION}

A PCB must have a stiff assembly that consists of various electronic equipment's like Integrated Circuits, passive and active elements, power electronic devices etc., The components are soldered or connected by wires together to make the PCB boards to perform a particular task. There are variety of PCB designs are available like single sided, double sided, 2D design, 3D printing etc., which depends upon the applications and cost of the PCB design [1]. In these above-mentioned methods, 3D PCB design has a vast area because of its novelty, less area of occupation, good physical structure etc., are

There are many software present, some of the most famous

- Altium Designer

- Autodesk Eagle

- KICAD EDA

- $\quad$ Solid work PCB

- OVR Stylus

- Morph Sensor

- Fusion 360

Some of them are free software and some of them require subscription. For example, Altium Designer is used commer- cially but not free. Morph sensor is a 3D electronic design software specially made for reforming sensor modules [2]. OVR Stylus is a 3D pen-based design software for virtual reality [3].

Fusion 360 originally is a CAD software used basically for designing mechanical and physical parts. In January 2020, Autodesk University (AU) has announced to combine the functionalities of Autodesk Eagle which is used for electronics designing. Since, Fusion 360 is a CAD modelling software it has a very big community support [4-5].

In real world it is necessary to use a particular software is various engineering fields like electrical, electronics, mechanical, civil for the purpose of designing a particular product. Most important reason to use Fusion 360 is we can move from an electronic CAD to mechanical CAD in just one data sheet. This cannot be possible in other PCB design software's. For manufacturing a complete product for example smartwatch, the model consists of both electronics and mechanical part.

Fusion 360 is the only software that provide to design both ECAD and MCAD in a single software. Besides it provides an easy user interface and supporting features that is require to build a complex multi-layer PCB [6]. In this paper a small 3D PCB is designed for protecting circuit by using Fusion 360 software.

\section{Designing PCB LAyout OF Protecting CiRCUit USING FUSION 360}

Before staring design process let us know about the circuit first. The first step to design a PCB is selection of components for the protection circuit. Because the small variation in size even in few millimeters need to change the entire circuit design. So, it is better to select the components accordingly before initiating the PCB design procedure.

The circuit we will be designing is protecting circuit using RT 1720 IC which is used for over voltage, over current, transient voltage and reverse polarity protection. It's a low-cost protection IC designed to simplify implementation and have wide Input Operation Range: $5 \mathrm{~V}$ to $80 \mathrm{~V}$ [7]. This protecting circuit can be used in multiple applications like mobile, laptop chargers, UPS charging systems, lab equipment's, industry apparatus's and other day to day smart electronic devices. After 
connecting all the component logically, the schematic diagram (fig. 1) is ready and the simulation is run once to check any error before going to next step.

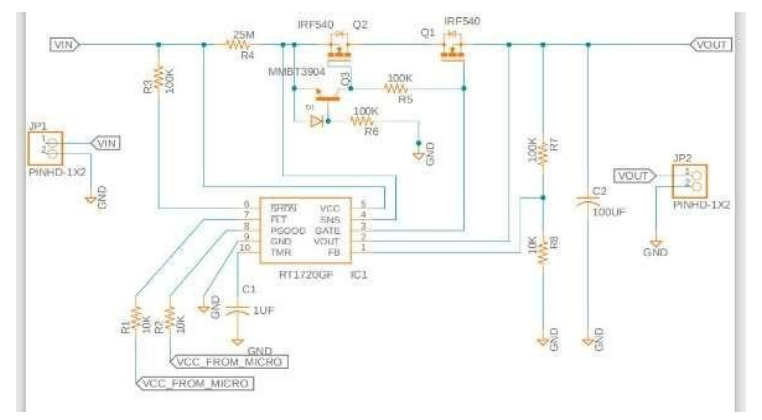

Fig. 1. Schematic Diagram

\section{PCB ROUTING}

It is most important step in designing the PCB. The first step is to align the components in the most efficient manner, this can be done by the move option in the toolbar. Then next step is routing, where the components are connected with each other and ground. Now a days, most of the design software are comes with auto-routing feature. This is a very tedious process in terms of 3D PCB design.

Auto-routing can reduce the circuit design complexity, time duration to design a PCB and also can be used to accommodate the components based on its size. Fusion 360 provides both manual and auto- routing feature. Once the DRC is complete, PCB layout (fig. 2) is complete.

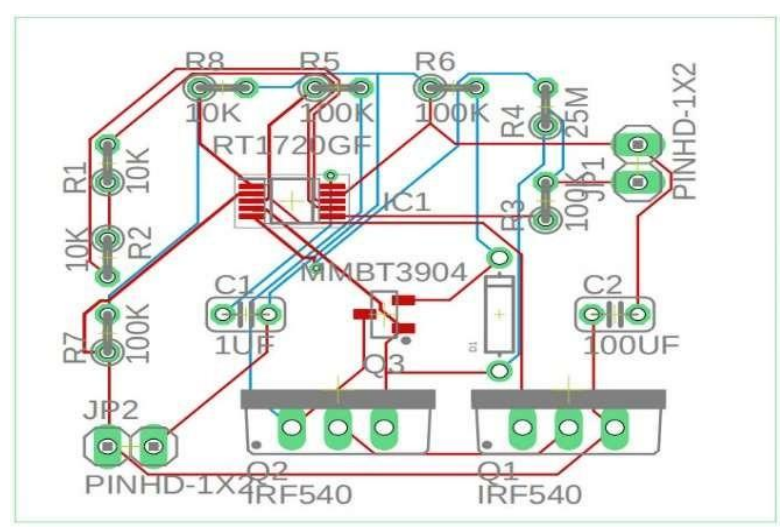

Fig. 2. PCB Routing

Once the routing is complete, the design tools are used again to create manufacturing drawings and the output files that the manufacturer will use to build the board. The design and manufacturing of a circuit board is a step-by-step process: schematic creation and simulation, setting up PCB design grids and DRCs, component placement, PCB routing, power planes, and finally assembling the BOM and building the board. Each process should be monitored carefully to get a successful design of a particular PCB.

The first step is the schematic creation which can be the basic one to route the components inside the PCB. Once the schematic for the proposed circuit is created, then the circuit will be simulated. The simulation analysis of every design is essential to avoid the faults like disconnection in a circuit, or short circuit between the components in the PCB.

After this simulation process, setting up of grids in PCB design has to be done. The grid design ensures that all electronic devices in that PCB are fixed in their own position virtually. The 3D PCB design needs more cooling surface rather than other conventional methods because of compact circuit design. This process can be also analyzed using the grid design procedure.

After grid design, the components need to be placed in their respective positions. This process should be start according to the size of the components from small to big size. Because of 3D printing, the level of the components should be exactly fixed to implement in PCB. Then routing can be done in PCB by following the same steps that has prescribed in the previous section. After this routing process it is possible to get the semifurnished PCB (only PCB without any major components) Then finally Bill of Materials (BOM) has to be assembled in the 3D printed PCB.

The CAM processor in Fusion 360 provides generating manufacturing files in just two clicks of a button, generating Gerber, drill and assembly files. Each file will have the prescribed details about the drilling size, depth and position in millimeter scale. This will help to place the components in their predefined position more accurately within very short duration. This process helps much in production by reducing the time consumption.

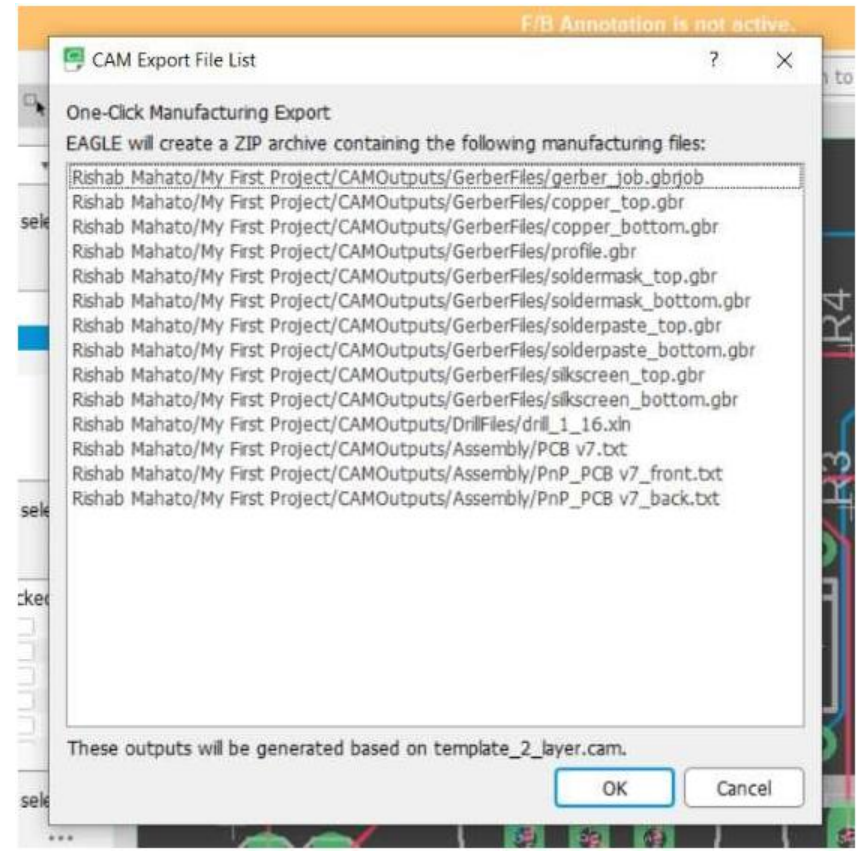

Fig. 3. Manufacturing files

\section{3D MODELING OF THE PCB LAYOUT}

In the PCB workspace, you can find the "View 3D PCB" and the "View 3D PCB with Canvas" commands. We can use either of them to create a 3D PCB (fig. 3). A 3D model is crated using STEP file which is a CAD file format, usually used to share 3D models between users with different CAD systems. 
Besides, Fusion 360 provides to design our own customize 3D model.

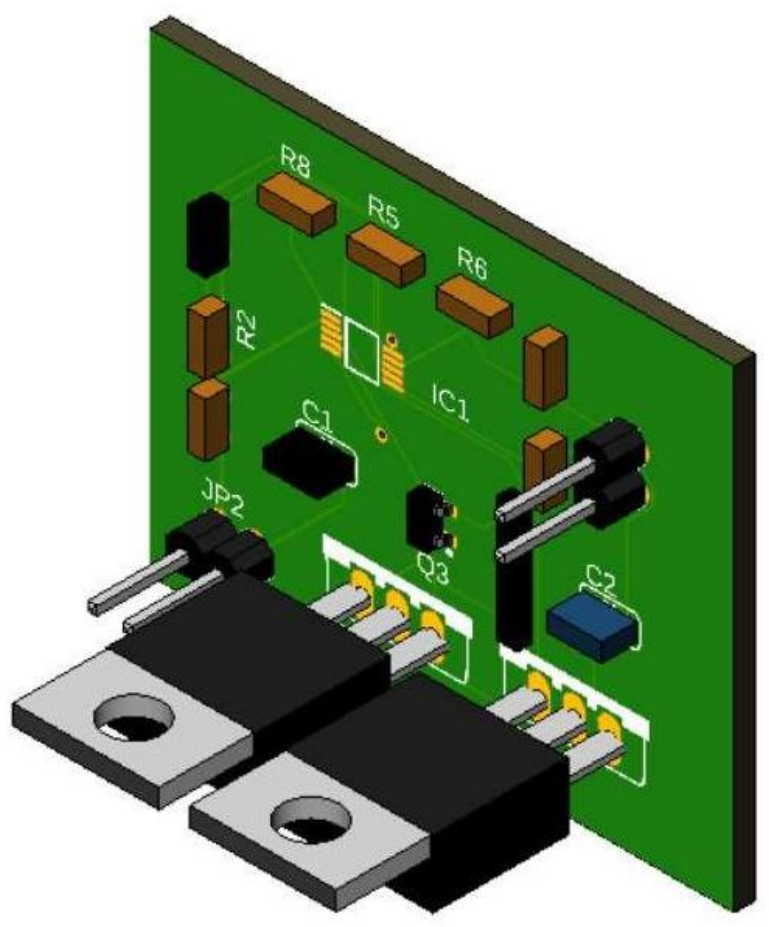

Fig. 4. 3D Model

As mentioned in the 3D model diagram, the physical structure of the PCB can be seen by virtually by using the Fusion 360 software. This will enhance the quality of manufacturing by previously determining the structure of the product. So, It can also worthful to design any type of $3 \mathrm{D}$ design by using this software.

\section{CONCLUSION}

PCB designing is a very important \& excellent stream in the field of Electronics. A size \& Beauty of a electronic product lies in the hand of a designer. It is possible to design, develop and test complete product using Fusion 360. A simulation model, and the structure of prototype is developed for a simple protecting circuit using Fusion 360. As India is developing in Electronics field Tremendously, there are very good scope \& future especially in such fields like 3D PCB designing.

\section{REFERENCES}

[1] Printed Circuit Board Introduction website [online]. Available on https://resources.pcb.cadence.com/blog/ 2019- printed- circuit- board-anintroduction

[2] Zhu, Junyi, Yunyi Zhu, Jiaming Cui, Leon Cheng, Jackson Snowden, Mark Chounlakone, Michael Wessely, and Stefanie Mueller. "MorphSensor: A 3D Electronic Design Tool for Reforming Sensor Modules." In Proceedings of the 33rd Annual ACM Symposium on User Interface Software and Technology, pp. 541-553. 2020.

[3] Jackson, Bret. "OVR Stylus: Designing Pen-Based 3D Input Devices for Virtual Reality." In 2020 IEEE Conference on Virtual Reality and 3D User Interfaces Abstracts and Workshops (VRW), pp. 13-18. IEEE, 2020.

[4] Autodesk Fusion 360 website [online]. Available on https://www.autodesk.in/products/fusion-360/features\# electronics

[5] Manufacturing Data website [online]. Available on https://www.pcb3d.com/tutorials

[6] More about Fusion 360 website [online]. Available on https://forums.autodesk.com/t5/fusion-360-electronics

[7] About the circuit website [online]. Available on https://circuitdigest.com /electronic-circuits/ 